\title{
Trailing-edge serrations: improving theoretical noise reduction models
}

\author{
Lorna J. Ayton* \\ University of Cambridge, Cambridge, UK CB3 OWA \\ Máté Szóke ${ }^{\dagger}$ and William Devenport ${ }^{\dagger}$ \\ Virginia Tech, Blacksburg, VA 24061, United States \\ Paruchuri Chaitanya ${ }^{\S}$ \\ University of Southampton, Southampton, UK SO17 1BJ
}

\begin{abstract}
This paper discusses the elements that make up a theoretical model for predicting the noise generated by a serrated trailing edge, and in particular, the required input to such a model; the wavenumber frequency spectra of the turbulence at the edge. It is proposed that this input, which is often modeled empirically, varies between a straight edge and a serrated edge and this fundamental difference in turbulence structure at the trailing edge leads to inaccurate theoretical predictions of noise reduction. Experimental measurements are therefore taken for straight and serrated trailing edges, with a particular focus on measuring the quantities which arise in typical wavenumber-frequency spectra models such as the TNO model. Whilst elements like the boundary layer thickness, shear profile, and skin friction velocity are found to be similar across the straight and serrated edges, the normal velocity wavenumber spectra is observed to vary substantially between the straight edge, and different locations along the serrated edge. In particular, the high-frequency decay rate of this spectra is reduced for a serrated edge versus a straight edge. This observed difference is then incorporated into a simple Chase-style empirical wavenumber-frequency spectrum; theoretical predictions which take account of a weaker high-frequency decay rate for a serrated edge agree much better with the experimental far-field noise predictions than theoretical predictions which assume an identical turbulent structure for both straight and serrated edges.
\end{abstract}

\section{Introduction}

Aerofoil trailing-edge noise, generated by boundary layer turbulence scattering off the trailing-edge of an aerofoil, contributes significantly to overall aviation noise levels and rotating fan noise levels. The silent flight of owls [1-3] has inspired many recent new designs which show good noise reduction with a promising approach being to introduce serrations along the span of the trailing edge.

Many studies have been conducted for trailing-edge serrations, concluding that they are thought to reduce noise by permitting a destructive interference between acoustic fields generated at different spatial locations along the edge [4-7], and by generating a scattered field which can consist largely of cut off modes [8] thus reducing the far-field acoustic propagation. Noise reductions of $\sim 10 \mathrm{~dB}$ have been achieved in experimental tests for carefully selected serration shapes [9]. Serrations have also been seen to be highly efficient for leading-edge noise reduction [10-12] and recent theoretical models [13] can accurately predict the sound generated by aerofoil-turbulence interaction (leading-edge noise) for a variety of different serration geometries, the most prevalent of which in the literature is the classical sawtooth serration. However, despite the theoretical approach for leading-edge noise and trailing-edge noise being very similar, agreement between the theoretical model and experimental measurements for trailing-edge noise reduction when the edge is serrated is poor.

To understand why this is the case, we must ask ourselves what is different physically between leading-edge noise generation and trailing-edge noise generation. Of course the wake at the trailing-edge may produce a significant tonal

\footnotetext{
*EPSRC Early Career Fellow, Department of Applied Mathematics and Theoretical Physics, AIAA Member. 1.j .ayton@damtp.cam . ac. uk

$\dagger$ Senior Research Associate, Crofton Department of Aerospace and Ocean Engineering, AIAA Member m.szoke@vt.edu

$\doteqdot$ Professor, Crofton Department of Aerospace and Ocean Engineering, AIAA Associate Fellow.

${ }^{\S}$ RAEng Research fellow and lecturer, Institute of Sound and Vibration Research, AIAA Member. ccp1m17@soton. ac.uk
} 
effect for cut-in serrations [14] which is not accounted for theoretically, however it is a broadband discrepancy that is observed. We hypothesise therefore that the key difference is that for leading-edge noise, the turbulence is known a-priori as it is carefully generated (in laboratory settings) by an upstream grid. At the trailing edge, the serrations themselves may affect the boundary layer structure and thus the turbulence which is scattered by the edge, as recently seen to be the case in a one-sided flow situation [15]. Indeed from [16] we see that by inputting a numerically determined surface pressure to the theoretical model [8], we recover a good agreement between the theoretically predicted far-field noise spectrum and the numerically calculated one. In this paper, we therefore attempt to quantify how trailing-edge sawtooth serrations affect the parameters which go into empirical turbulence models used by theoretical predictions.

\section{Analytical Model}

Here we first briefly review the analytical model for predicting trailing-edge noise, as presented in [8].

A wall-normal gust with pressure

$$
p_{i}=P_{i} e^{i\left(k_{1} x+k_{3} z-\omega t\right)},
$$

is scattered by a semi-infinite plate lying in the region $x<\tilde{c} F(z), y=0$, with $x$ denoting the streamwise direction, $z$ the spanwise direction, and $y$ the normal direction. A uniform flow with Mach number $M$ is assumed. $F(z)$ denotes the periodic serration shape, normalised such that $F_{\max }-F_{\min }=0.5$. The parameter $\tilde{c}$ then permits a variable 'tip-to-root' ratio, i.e. a varying difference of the height of the trailing-edge geometry.

The scattered pressure, $p e^{-i \omega t}$, satisfies the compressible convective Helmholtz equation,

$$
\beta^{2} \frac{\partial^{2} p}{\partial x^{2}}+\frac{\partial^{2} p}{\partial y^{2}}+\frac{\partial^{2} p}{\partial z^{2}}+2 i k M \frac{\partial p}{\partial x}+k^{2} p=0
$$

subject to boundary conditions of zero normal velocity on the rigid surface

$$
\left.\frac{\partial p}{\partial y}\right|_{y=0}=0 \quad x<\tilde{c} F(z)
$$

and zero pressure jump across the wake downstream of the plate

$$
\left.\Delta p\right|_{y=0}=-e^{i\left(k_{1} x+k_{3} z\right)} \quad x>\tilde{c} F(z),
$$

where $\Delta$ denotes the jump in value across $y=0_{+}$and $y=0_{-}$. Here $\beta^{2}=1-M^{2}$ and $k=\omega / c_{0}$, with $c_{0}$ the speed of sound. We suppose the wake consists of a flat vortex sheet along $y=0, x>\tilde{c} F(z)$.

Since the geometry is periodic in the spanwise direction we impose a spanwise periodicity condition for the solution;

$$
\left.p\right|_{z=1}=\left.p\right|_{z=0} e^{i k_{3}},\left.\quad \frac{\partial p}{\partial z}\right|_{z=1}=\left.\frac{\partial p}{\partial z}\right|_{z=0} e^{i k_{3}}
$$

where lengths have been non-dimensionalised by the serration wavelength, thus the period in the spanwise direction is 1 .

This mixed boundary condition problem is solved via application of the Wiener-Hopf technique following a transformation of coordinates to

$$
\begin{aligned}
& \xi=\frac{x}{\beta}-c F(z), \\
& \eta=y, \\
& \zeta=z,
\end{aligned}
$$

where $c=\tilde{c} / \beta$.

The far-field scattered pressure (full details of the derivation may be found in [8]) in cylindrical polar coordinates $(r, \theta, z)$ centred on the trailing edge is then given by

$$
p(r, \theta, z) \sim \sum_{n=-\infty}^{\infty} \frac{E_{n}\left(-w_{n} \cos \theta\right) \sqrt{-\delta-w_{n}}}{2 i\left(\delta-w_{n} \cos \theta\right)} Z_{n}\left(-w_{n} \cos \theta, z\right) \sin \left(\frac{\theta}{2}\right) \frac{e^{i w_{n} r} e^{i \pi / 4}}{\sqrt{\pi r}} e^{i w_{n} \cos \theta c F(z)} e^{-i k M x / \beta^{2}},
$$

where $\delta=\tilde{k}_{1} \beta$, with $\tilde{k_{1}}=k_{1}+k M \beta^{-2}$,

$$
Z_{n}(\lambda, \zeta)=e^{-i \lambda c F(\zeta)} e^{-i k_{3} \zeta+2 n \pi i \zeta},
$$




$$
w_{n}^{2}=\left(\frac{k}{\beta}\right)^{2}-\left(k_{3}+2 n \pi\right)^{2}
$$

and

$$
E_{n}(\lambda)=\int_{0}^{1} e^{i \tilde{k}_{1} c F(\zeta)+i k_{3} \zeta} \bar{Z}_{n}(\bar{\lambda}, \zeta) d \zeta
$$

No assumptions thus far have been made over the boundary layer turbulence except that it may be decomposed into wall-normal gusts. To account for a realistic turbulent boundary layer, we must indicate which gusts are permitted, in the form of a turbulent spectrum. As such, the total far-field sound spectra due to a fully turbulent boundary layer is given by

$$
\operatorname{PSD}\left(k_{1}, \omega\right)=10 \log _{10}\left(\int_{-\infty}^{\infty}|p|^{2} \Pi\left(\omega, k_{1}, k_{3}\right) d k_{3}\right),
$$

where $\Pi\left(\omega, k_{1}, k_{3}\right)$ is the wavenumber-frequency spectra of the turbulence on the plate. It is this function that is unknown, and theoretical or semi-theoretical predictions can vary from using a simplistic approximation as given by [17] based on Chase's model [18];

$$
\Pi \sim \frac{4 C_{m} \rho_{0}^{2} v_{*}^{4}\left(\omega / U_{c}\right)^{2} \delta^{4}}{U_{c}\left(\left(\left(\omega / U_{c}\right)^{2}+k_{3}^{2}\right) \delta^{2}+\chi^{2}\right)^{2}},
$$

to a complex definition [23]

$$
\Pi=4 \rho_{0}^{2} \int_{0}^{\infty} \frac{k_{1}^{2}}{\kappa^{2}}\left(\frac{d U}{d y}\right)^{2} \Lambda_{2}(y) \phi_{22}\left(k_{1}, k_{3}, y\right) e^{-2 \kappa y} d y
$$

where in both cases the approximation $k_{1} \approx \omega / U_{c}$ has been applied, where $U_{c}=\alpha U$ is the convection velocity in the boundary layer. Further, $\delta$ is the boundary layer thickness, $\rho_{0}$ the air density, $v_{*}$ the friction velocity nondimensionalised by the mean flow, $\Lambda_{2}$ is the vertical integral lengthscale of the turbulence, $\kappa=|\vec{k}|$, and $\phi_{22}\left(k_{1}, k_{3}, y\right)$ is the wavenumber-frequency spectrum of the vertical velocity fluctuations. The parameters $\chi=1.33$ and $C_{m}=0.1553$ are non-dimensional parameters fitted to data. These models however are determined for a straight edge, and do not account for any effects of the serrations on the turbulent structure itself.

It is this influence of serrations on the boundary layer turbulence which we will interrogate here, through assessing experimental data collected both in the far field and on the surface of serrations. For the improvement of a turbulent spectra model, We specifically aim to measure parameters associated with both simple and complex functional expressions of the wavenumber-frequency spectra on the surface of the serrations close to the trailing edge, to understand what effects the serrations themselves have on the validity of such functions.

\section{Experimental Setup}

Experiments have been conducted in the Stability Wind Tunnel at Virginia Tech, during April 2020. They focused on capturing the far-field trailing edge noise generated by various serration geometries. In addition, measuring the surface pressure fluctuations along the edge of the serration geometries is at the basis of validating the analytical model proposed in Section II

The schematic of the measurement setup is shown in Fig. 1. The aerofoil used in the current study is a NACA0012 aerofoil with a $3-\mathrm{ft}$ chord and a 6 -ft span. The aerofoil was set to a $0^{\circ}$ geometrical angle of attack and the serrations proposed in the current study were attached to the trailing edge. The free-stream flow speed was set to 40,50 and 60 $\mathrm{m} / \mathrm{s}$, resulting in Reynolds numbers of 2.5, 3.0, 3.5 million, respectively. The serrations were made using a Stratasys Connex 3 rapid prototyping machine and they were attached to the NACA0012 aerofoil, which is made of fiberglass composite material, see Fig. 1 .

The trailing edge noise in the far-field (anechoic chamber) was captured using a beamforming array, built by AVEC Inc, consisting of 251 G.R.A.S. 40PH-S5 type microphones. In addition, the flow field in the vicinity of the trailing edge has been measured using a LaVision high-speed PIV system.

\section{The Virginia Tech Stability Wind Tunnel}

The Stability Tunnel is a low-speed closed circuit wind tunnel used for a mix of commercial testing, research and educational experiments on the campus of Virginia Tech in Blacksburg, Virginia [19, 20]. The facility, driven by a 


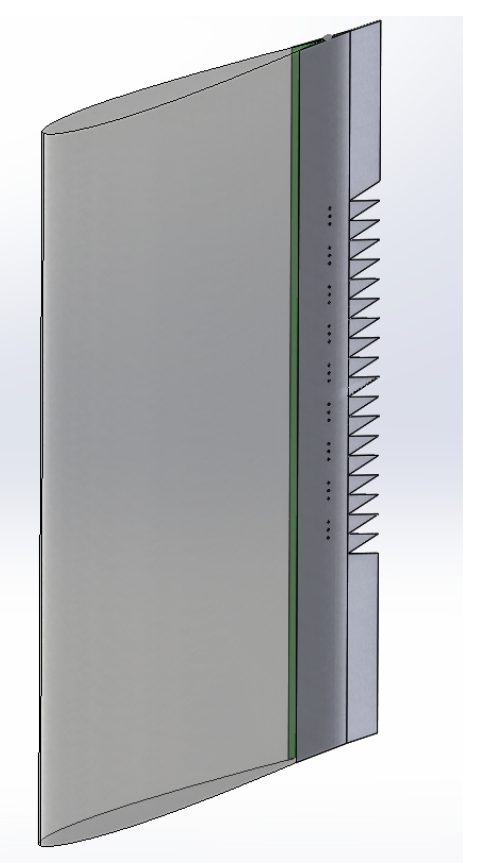

Fig. 1 The CAD view of the NACA0012 aerofoil with the add-on serrations.

$600 \mathrm{HP}$ fan, can generate speeds of up to $80 \mathrm{~m} / \mathrm{s}$ through its $7.3-\mathrm{m}$ long, $1.85-\mathrm{m}$ square test section. Flow through the empty test section is closely uniform and of very low turbulence intensity, increasing from $0.021 \%$ at $21 \mathrm{~m} / \mathrm{s}$ to $0.031 \%$ at $57 \mathrm{~m} / \mathrm{s}$. Two test section configurations are available. The hard wall "aerodynamic" test section configuration has walls formed from a sequence of aluminum panels. The Kevlar-wall aeroacoustic test section configuration used in the present work replicates the same interior dimensions as the hard wall test section configuration. The side walls of this test section, however, include 4.2-m long openings that span the full $1.85-\mathrm{m}$ test section height. These openings are covered using tensioned Kevlar cloth that is almost transparent to sound and only slightly porous to the flow. Sound generated by models in the test section travels out through these acoustic windows into the anechoic chambers that flank the test section. Most acoustic measurements are made using microphones placed in these chambers. Kevlar 120 cloth is used for the windows. This is a scrim made from a plain weave of Kevlar 49 fiber with 13.4 threads per centimeter in both warp and weft directions. For the tests reported here, cloth provided by EAS Fiberglas Company was used with an open area ratio of $1.8 \%$, weight of $58 \mathrm{~g}$ per square meter, thickness of $0.08 \mathrm{~mm}$, and equivalent membrane thickness of $0.021 \mathrm{~mm}$. The Kevlar forming the acoustic windows is supported on tensioning frames under a nominal no-flow tension of $1500 \mathrm{~N} / \mathrm{m}$. The ceiling and floor of the acoustic test section are formed, primarily, from perforated metal panels backed by $0.46-\mathrm{m}$ acoustic wedges to serve as sound absorbers. The perforated panels are covered with additional Kevlar cloth in order to form a low-noise flow surface. Two-dimensional airfoil models are mounted in the same position as in the hard wall test section and identical angle of attack control and sensing systems are used. Floor and ceiling panels in the immediate vicinity of the model are also replaced with impermeable aluminum panels so as to avoid flow through the acoustic treatment driven by model-generated pressure gradients.

\section{Serration Geometry}

Three serration geometries were assessed in the experimental campaign. The geometry of the serrations is shown in Fig. 3 The wavelength of the geometry has been kept constant $(\lambda=5 \mathrm{~cm})$ and the tip-to-root height of the serrations $(2 h)$ were be set to 5,10 , and $15 \mathrm{~cm}$. In the following discussions, these geometries are referred to as Case 1, Case 3, and Case 3, respectively. These are compared to a baseline (no serration) case where a 10cm straight-edged insert is appended to the aerofoil. 


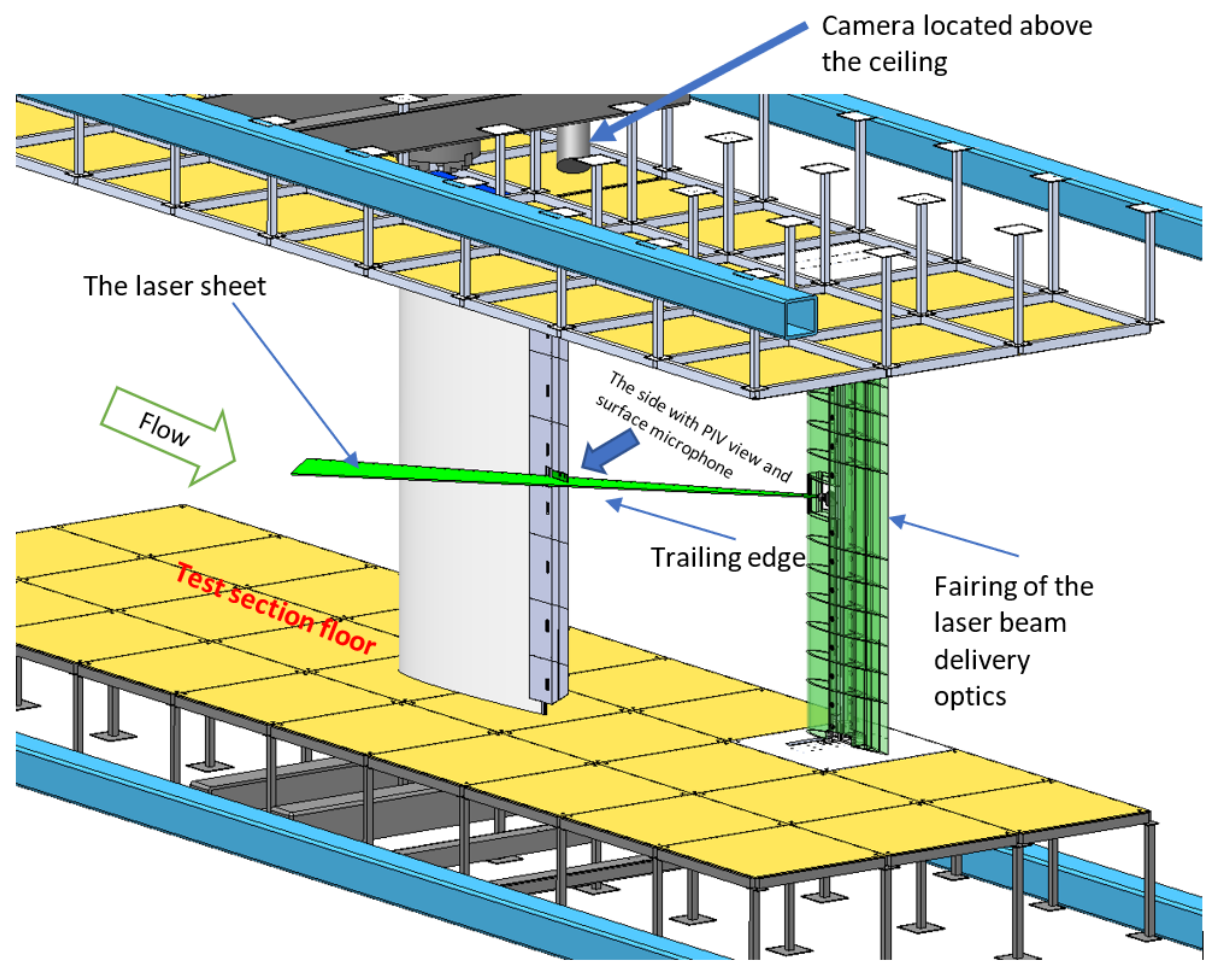

Fig. 2 The CAD view of the NACA0012 aerofoil within the Stability Wind Tunnel.

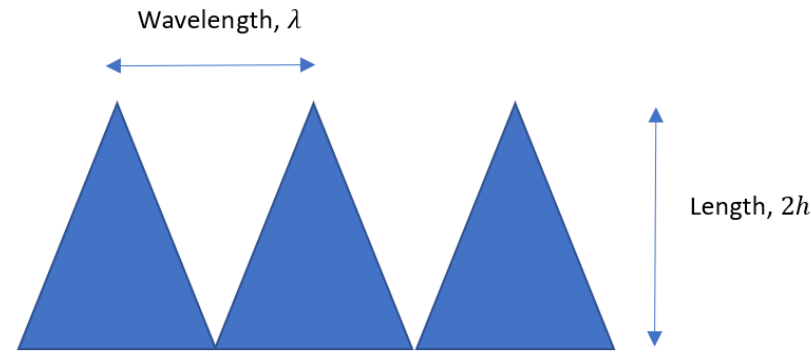

Fig. 3 The geometry of the serrations proposed in the current study. 


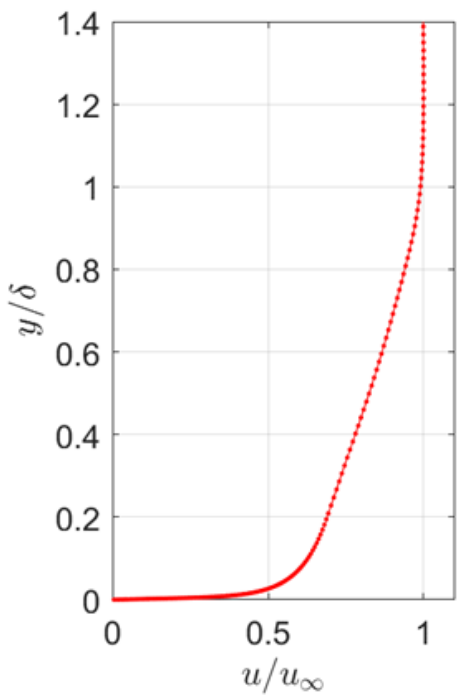

Fig. 4 Boundary layer velocity profile at $40 \mathrm{~m} / \mathrm{s}$ freestream velocity for the baseline case.

\section{Velocity Field in the Close Vicinity of the Serrations}

The velocity field over the serrations was measured using a time-resolved stereo particle image velocimetry system (TR sPIV). The system, developed by LaVision, consists of a high-speed Phantom v2512 camera and a Photonics Industries DM150-532-DH type Nd:YAG dual head high-speed laser. The PIV assembly offers the measurement of a $2 \mathrm{D}$ velocity field within a $1.5 \mathrm{~mm}$ thick LASER sheet at a $24 \mathrm{kFrames} / \mathrm{s}$ sampling rate, resulting in a maximum of $12 \mathrm{kSamples} / \mathrm{s}$ of velocity field data. The schematic view of the optical arrangement for the PIV measurement is shown in Fig. 2. The camera was positioned at a 4-ft distance from the mid-span of the trailing edge, and by using a Nikon $300 \mathrm{~mm} / \mathrm{F} 4$ lens. After applying a 16-pix-by-16-pix interrogation window, the spatial resolution of the PIV data was $1.5 \mathrm{~mm}$ times $1.5 \mathrm{~mm}$. The laser sheet was introduced to the test section from downstream of the model. The laser optical system was embedded into a NACA0018-shaped airfoil profile with a 1.6-ft chord, see Fig. 2. This resulted in a one-side biased blockage of the test section, which was observed as a constant angle-of-attack offset of the airfoil condition. After quantifying the lift curve of the airfoil with and without the laser-optical stand, it was found that the optical stand generated a constant 0.5 degree offset in the aerodynamic performance of the NACA0012 airfoil.

Our aim is to utilize the TR sPIV for the measurement of the velocity field over the edge of the serrations at two locations, namely, at midpoint and at the root of the serrations edge. In addition, the far-field noise using a 251-element far-field beamforming array were measured simultaneously alongside the velocity field. This approach opens the opportunity to find links between the developing velocity field and both the surface pressure fluctuations and to the far-field noise. Utilizing the high sampling frequency offered by TR sPIV system, we expect to gain a better insight to the generation of trailing-edge noise not only from the acoustic aspect but also from the turbulence point of view.

\section{Results}

\section{A. Baseline results}

In this section, we present a set of results for the baseline case (straight edge). Figure 4 presents the mean velocity profile within the turbulent boundary layer over the trailing edge of the NACA0012 airfoil. Figure 5 presents the velocity profile non-dimensionalized using the inner-layer scales, namely, $u^{+}=u(y) / u_{\tau}$ and $y^{+}=y u_{\tau} / v$ with $u_{\tau}$ being the friction velocity at the wall and $v$ is the viscosity of air at the test conditions. The friction velocity was found by iteratively fitting the measured curve to the law of the wall while minimizing the discrepancy between the two curves. From this, the boundary layer was extrapolated below the lowest wall-normal $(y)$ point obtained from the PIV data. This is shown in Figure 4. 


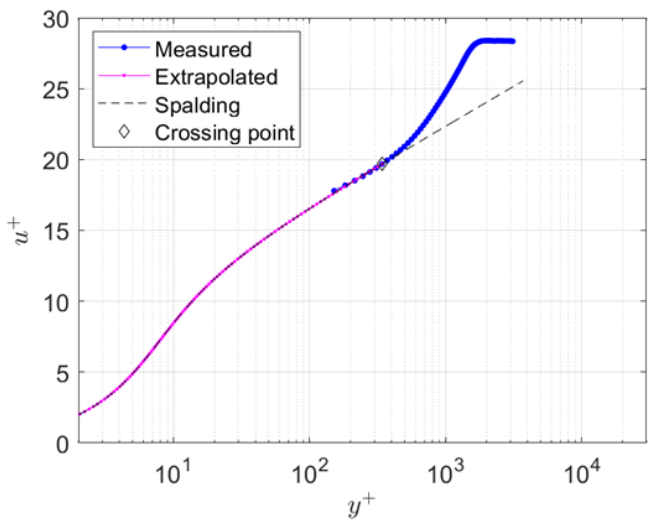

Fig. 5 Boundary layer velocity profile compared to the law of the wall at $40 \mathrm{~m} / \mathrm{s}$ freestream velocity for the baseline case.

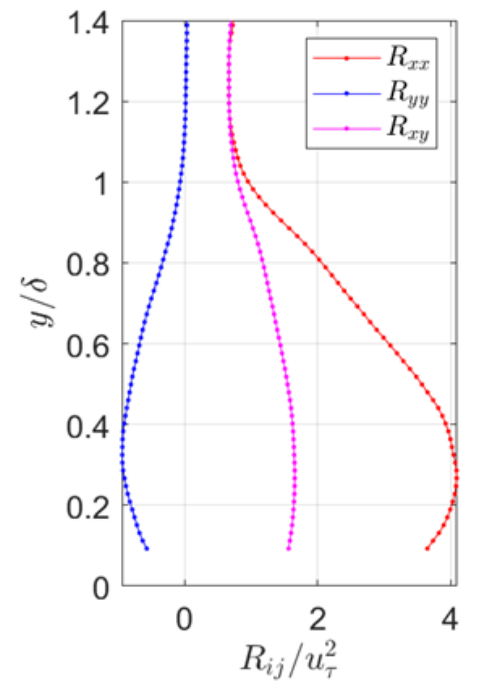

Fig. 6 Streamwise-averaged Reynolds shear stress profiled obtained at $40 \mathrm{~m} / \mathrm{s}$ freestream velocity for the baseline case. 

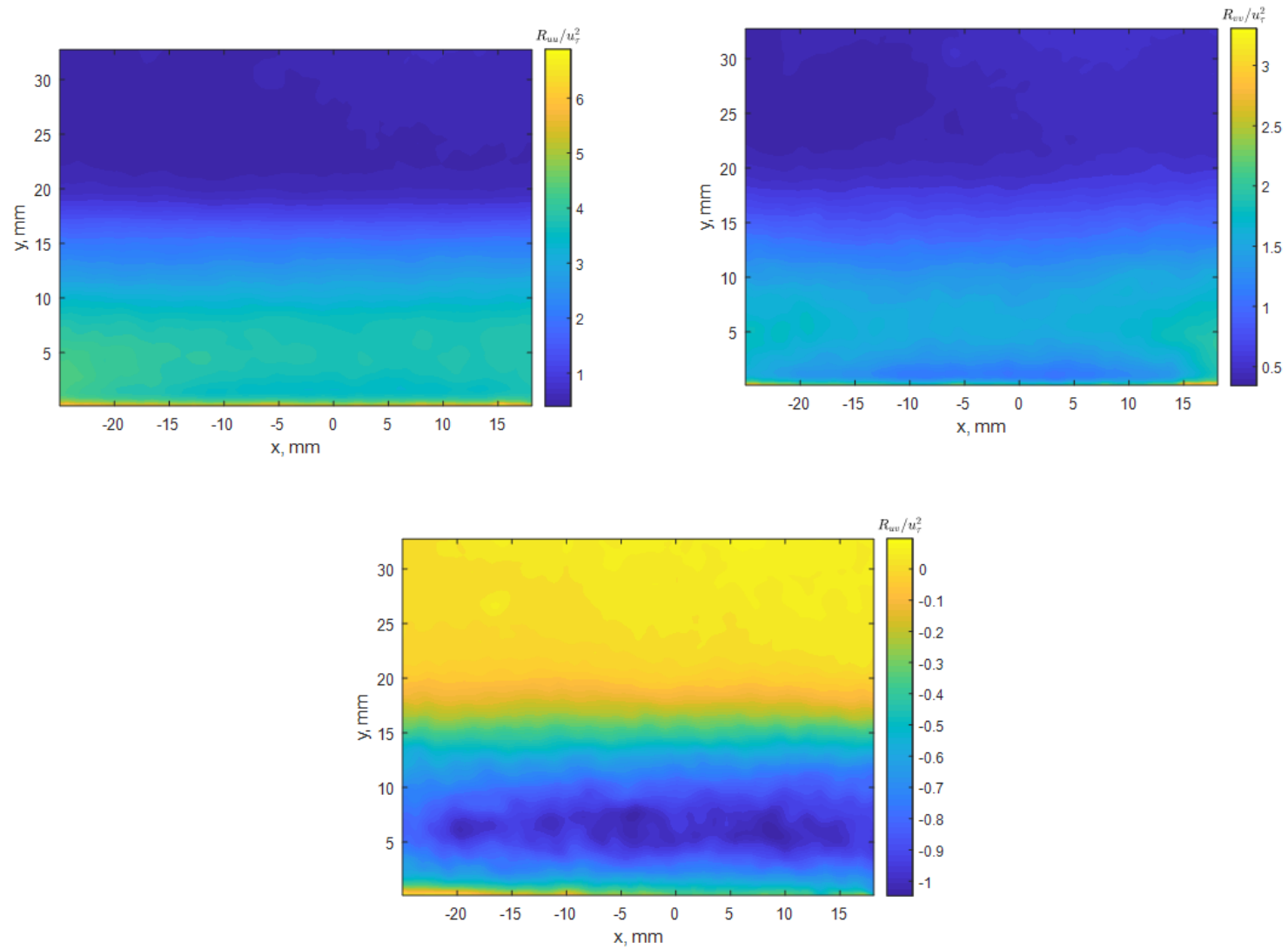

Fig. 7 Reynolds shear stress contour maps $\left(R_{u u}, R_{v v}\right.$, and $\left.R_{u v}\right)$ obtained at $40 \mathrm{~m} / \mathrm{s}$ freestream velocity for the baseline case, $x=0 \mathrm{~mm}$ denotes the trailing edge. 

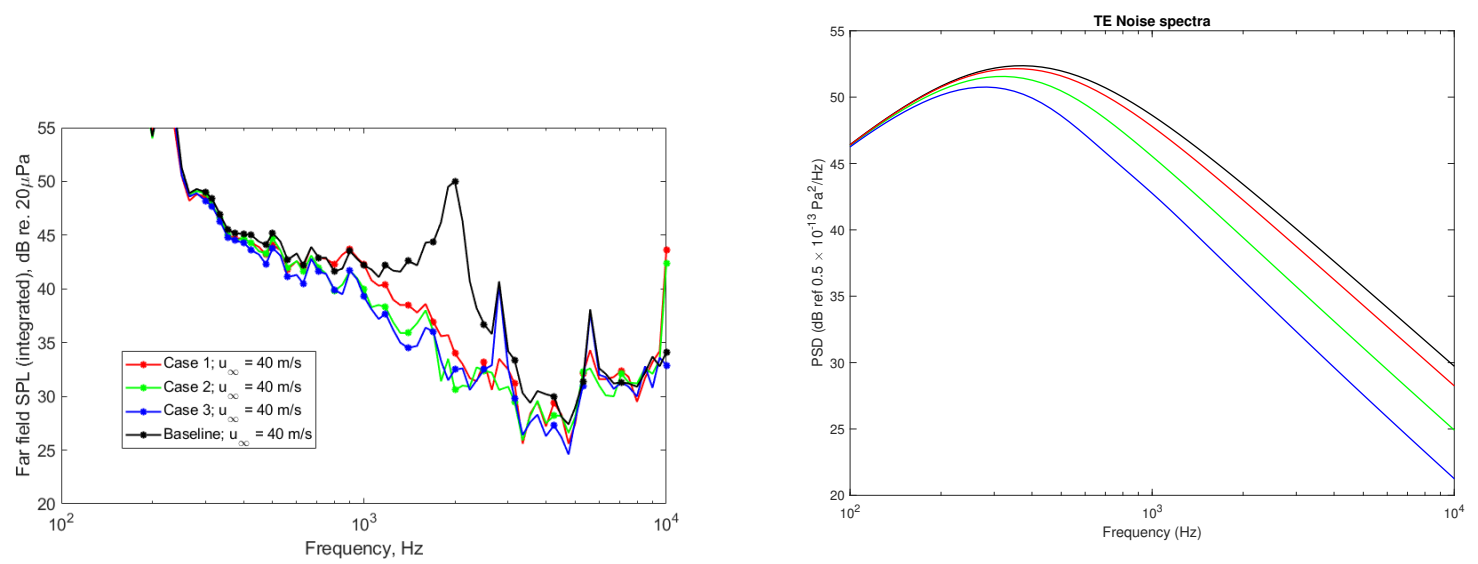

Fig. 8 Left: integrated far-field noise SPL levels around the trailing edge of the serrations at $40 \mathrm{~m} / \mathrm{s}$ freestream velocity. Right: Theoretical predictions using the Chase spectrum.

\section{B. Far-field noise}

The far-field noise sound pressure levels are shown in Figure 8 alongside theoretical predictions obtained using the simple Chase-type spectrum. The presented experimental SPLs were obtained by integrating the beamforming maps over a window covering the trailing edge of the serrations. The tonal noise observed for the baseline case is associated with the vortex-shedding frequency of the baseline case, as the 3D printed add-ons investigated here had a thickness of $3 \mathrm{~mm}$ to enable microphones being embedded in them. The vortex shedding is not present for the serrations case, as their trailing edge was inclined with respect to the flow. Whilst the vortex shedding is significant in the far-field noise results, it was not observed in the boundary layer data and surface wavenumber-frequency spectrum which are of primary concern in this paper.

We compare the measured far-field noise to that of predicted analytically when an identical Chase-type model is used for both the baseline and serrated cases. We see that whilst the overall trends are similar as the serrations vary, yet the overall noise reduction is overpredicted theoretically, and the reduction does not diminish at the highest frequencies which is observed in the data.

\section{Effect of serrations on the near-field}

Here we shall focus on the results calculated from the measured data for the Baseline case, and in two planes (root and half-edge) for the serrated Case 2. We are primarily interested in assessing features which arise in models for wavenumber-frequency spectra, namely the boundary layer profile, $U(y)$, convection velocity, $U_{c}$, boundary layer thickness, $\delta$, and friction velocity, $v_{*}$, which all appear in the simple Chase-type theoretical model, along with the vertical turbulence lengthscale, $\Lambda_{2}$, and (vertical) velocity wavenumber-frequency spectra, $\phi_{22}$, which are present in the more complex TNO-style model.

First we address the typical assumption, $k_{1}=\omega / U_{c}$, which is commonplace in most theoretical models. In Fig 9 we present the wavenumber-frequency spectra, and in the boundary layer profiles for Case 2 are compared against the baseline in Fig11 for measurements at the half-edge and root of the serration. We note overall the wavenumber-frequency spectra appear to behave similarly across the three situations, and is peaked in the convective domain, thus we postulate that the convection velocity of the dominant wavenumber. This is confirmed in Figure 10 where a reference line of gradient 4.6 is overlaid in each case, predicting $U_{c} \approx 28.9 \mathrm{~ms}^{-1}=0.72 U_{\infty}$.

Next we consider the boundary layer profiles in Fig 11 . Here too there is only a minor difference to the overall profile, and only a slight variation in boundary layer thickness for the three cases; baseline $(\delta=0.0182 \mathrm{~m})$, Case 2 root $(\delta=0.0228 \mathrm{~m})$ and Case 2 half edge $(\delta=0.0165 \mathrm{~m})$.

We next turn to the friction velocity, which measured at $1.37 \mathrm{~ms}^{-1}, 1.29 \mathrm{~ms}^{-1}$, and $1.36 \mathrm{~ms}^{-1}$ for baseline, case 2 (root) and case 2 (half) respectively yielding $v_{*}=0.034,0.032,0.034$ respectively which are negligibly different.

This has completed a comparison of the physical terms present in a simple Chase-type empirical model, with the conclusion that there is no significant difference in the parameters, and such changes to these parameters do not have a noticeable effect on the theoretical predictions. Nevertheless, the amplitude of the wavenumber-frequency spectra has 

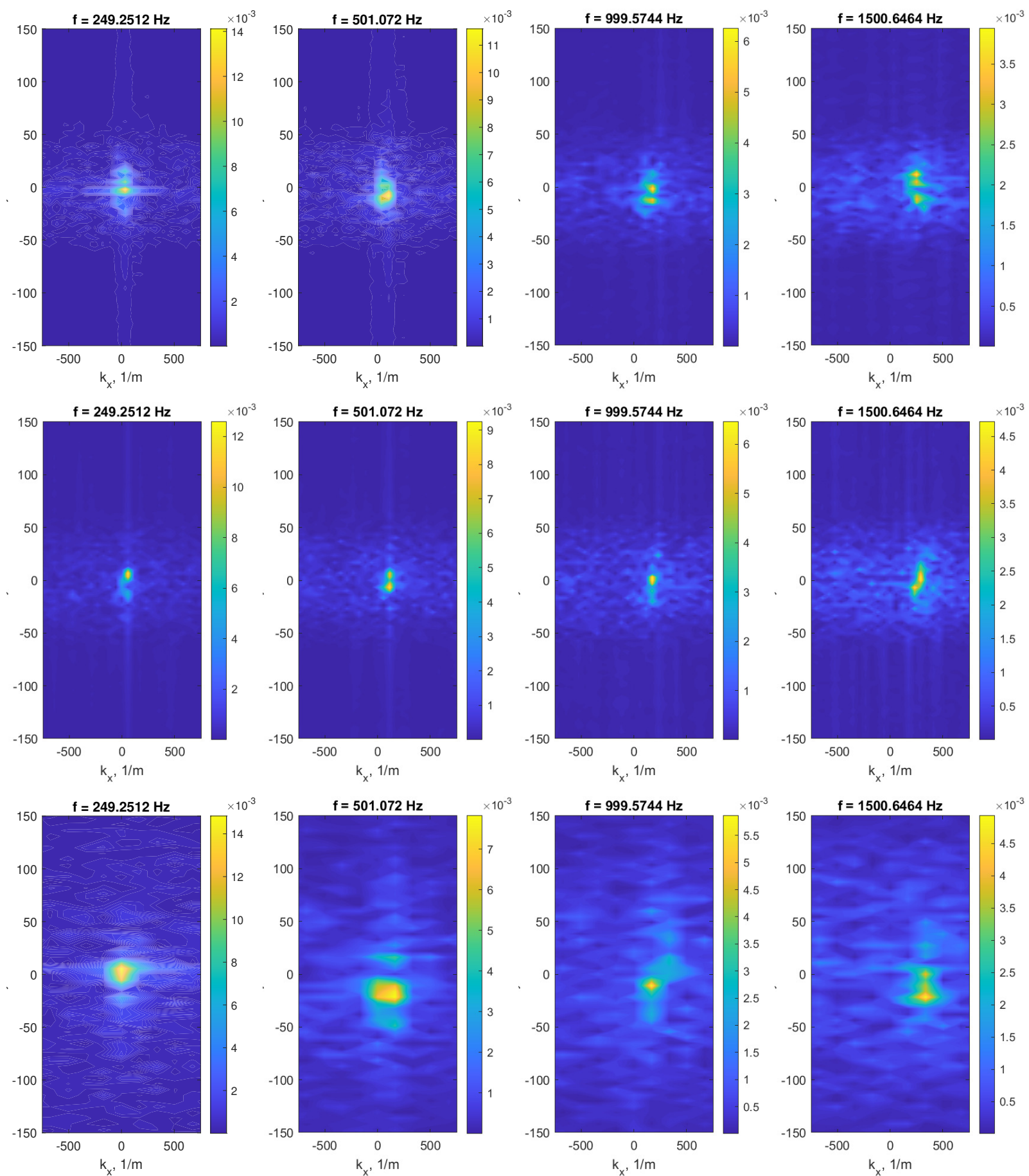

Fig. $9 k_{x}-k_{y}$ wavenumber frequency spectra for baseline (top), Case 2 half edge (middle) and Case 2 root (bottom). 

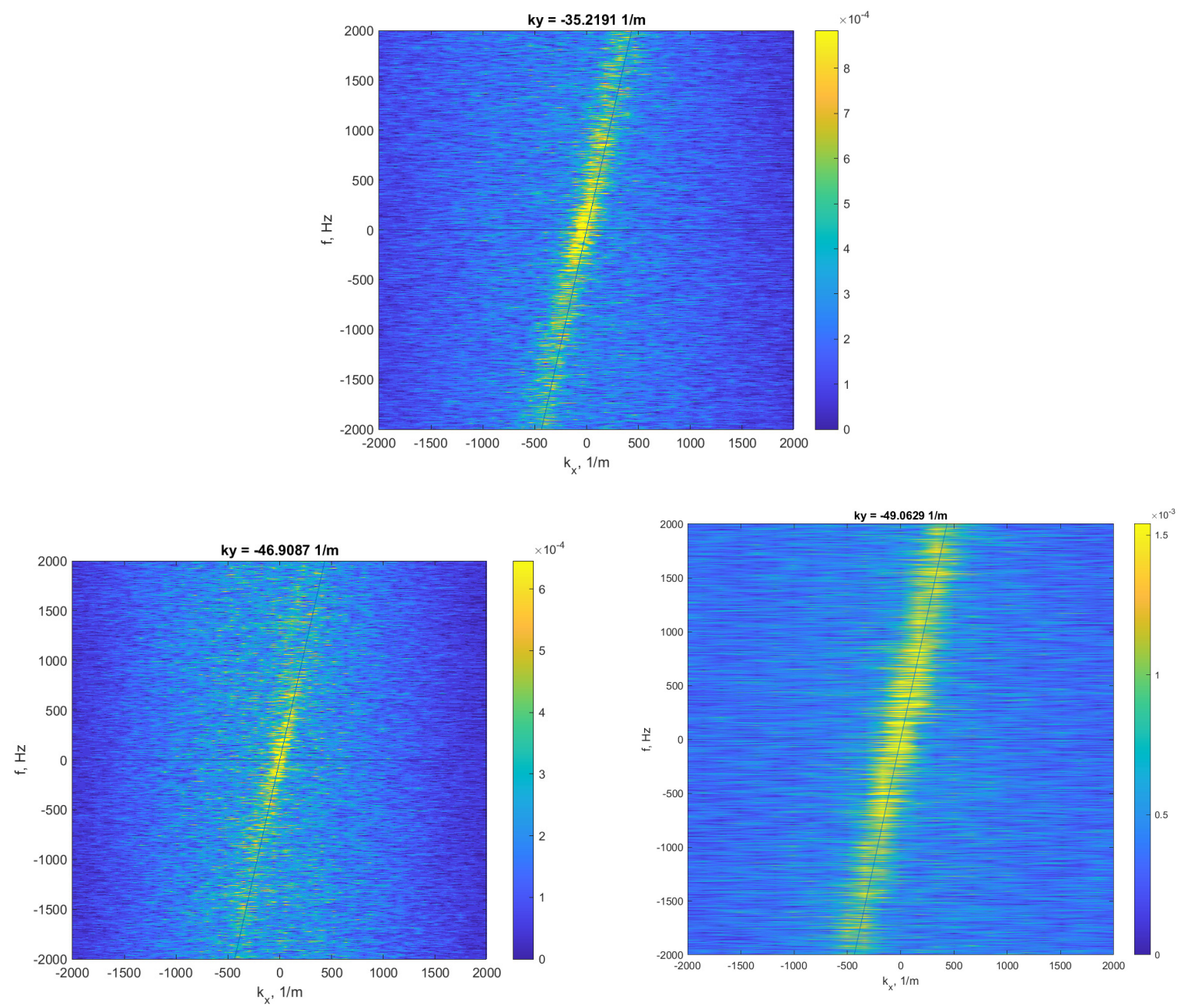

Fig. $10 k_{x}-\omega$ wavenumber-frequency spectra for baseline (top), Case 2 half edge (bottom left) and Case 2 root (bottom right).

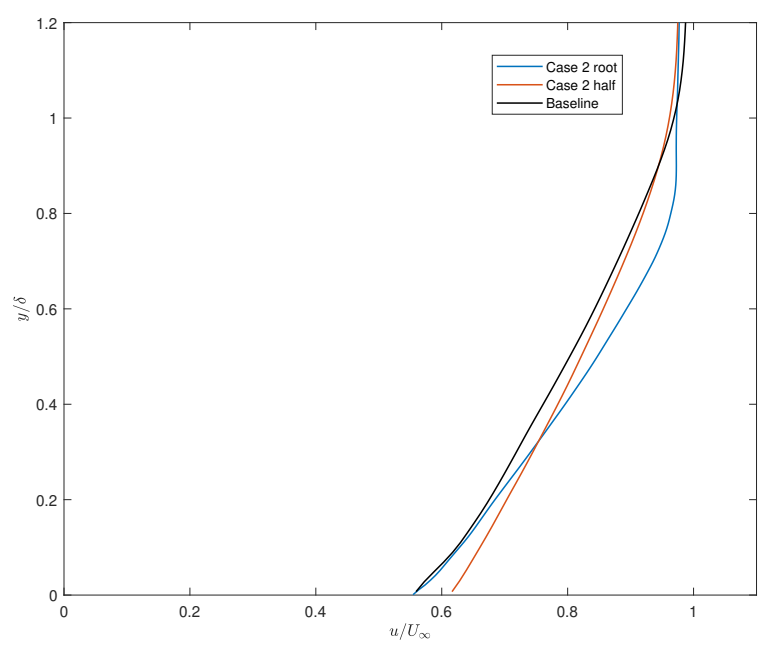

Fig. 11 Boundary layer profiles for two locations of Case 2 and the Baseline (straight) edge. 


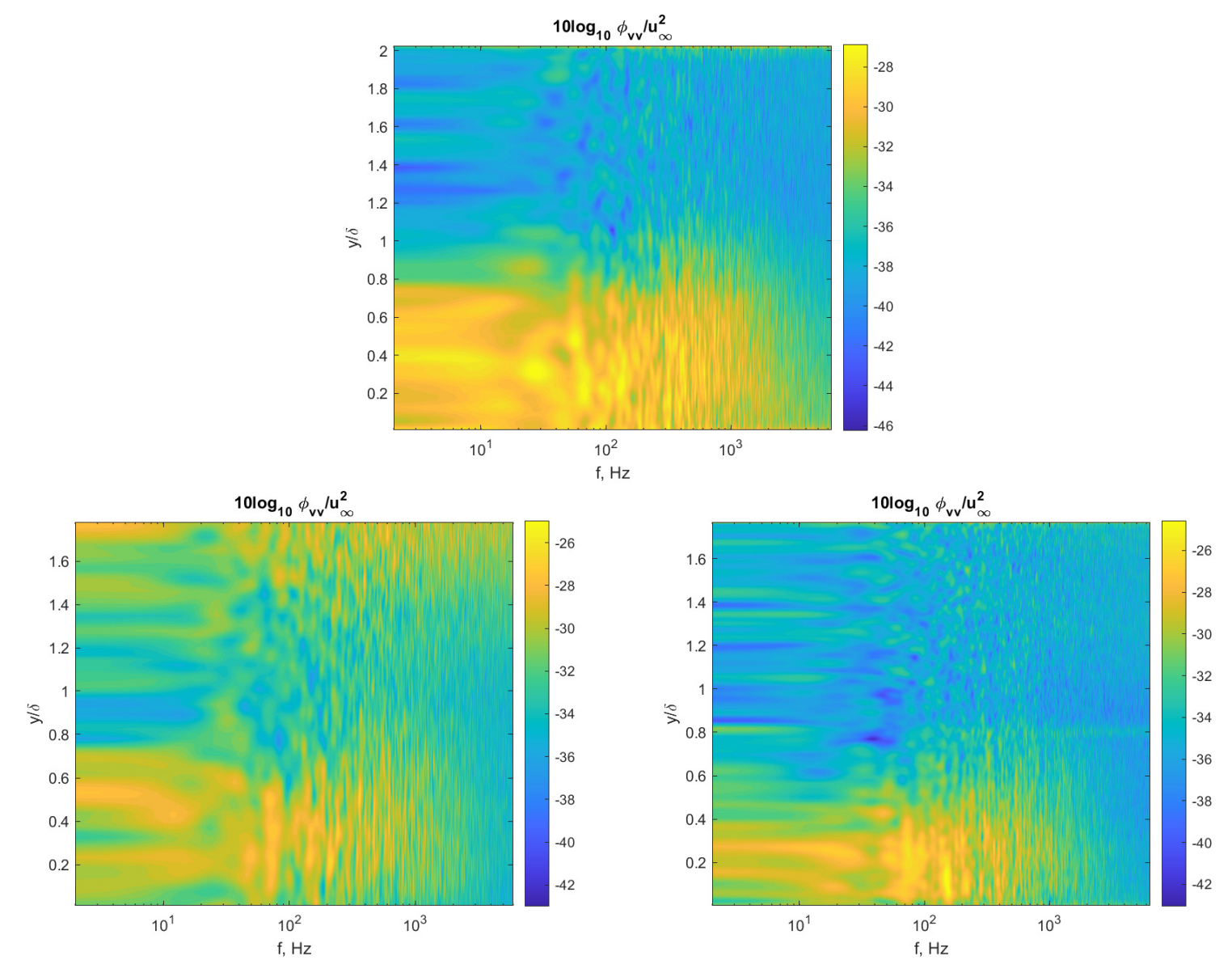

Fig. 12 Vertical velocity power spectral density $\left(\phi_{v v}(f, y)\right)$ for baseline (top), Case 2 half edge (bottom left) and Case 2 root (bottom right).

been seen to differ, implying the 'experimentally determined' $C_{m}$ of $\chi$, parameters could be dependent on serration geometry, or indeed the overall decay power on the denominator of the function may be affected by the serrations. Indeed, recent results from [15], albeit for one-sided flow, indicate the surface frequency spectrum does vary at the root and tip of a serration with the high-frequency decay rate being a point of difference, and these differ further from a baseline straight edge.

This potential variation in decay rate would also be reflected in the more complex TNO-style model, and will be dependent on the normal-velocity fluctuations at the edge. We consider this by plotting $\phi_{v v}(f, y)$, the measured vertical velocity power spectral density at the trailing edge, in Figure 12 for the baseline, and Case 2 root and half-edge locations.

The average result across all $y$ values is further given in Figure 13 directly comparing the baseline with the root and half edge of a serration ${ }^{*}$. There is a clear variation in moderate and high-frequency behaviour indicating indeed an alternate high-frequency decay rate is present at different locations of the serration. In particular, the serrated cases decay less rapidly with frequency than the baseline case, which would result in a lower noise reduction at high-frequencies than if the same spectra were used in a theoretical model.

We can implement this observation into the simple Chase-type model as follows

$$
\Pi \sim \frac{4 C_{m} \rho_{0}^{2} v_{*}^{4}\left(\omega / U_{c}\right)^{2} \delta^{4}}{U_{c}\left(\left(\left(\omega / U_{c}\right)^{2}+k_{3}^{2}\right) \delta^{2}+\chi^{2}\right)^{a}},
$$

where $a$ will determine the high-frequency decay rate. Selecting $a=2$ for the baseline is in line with a $f^{-1}$ scaling of

*The half-edge case have been shifted by $2.5 \mathrm{~dB}$ to ensure agreement with the other measurements at low frequencies - we believe the discrepancy in the data magnitude is a simple calibration error. 


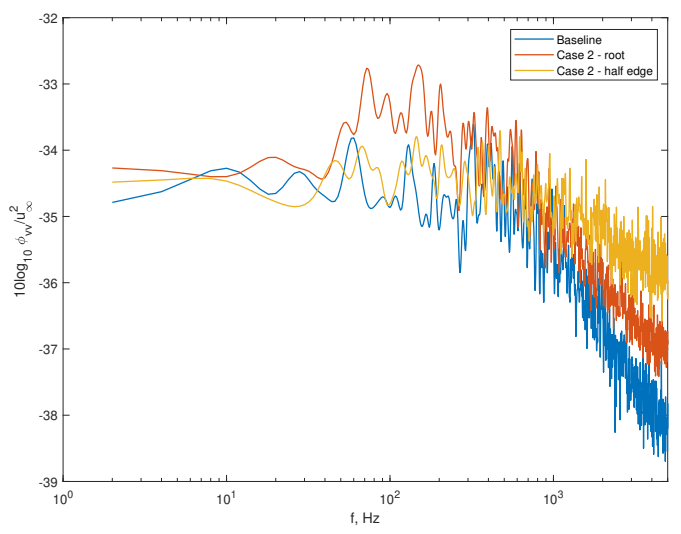

Fig. $13 \phi_{v v}(f)$ averaged over $y$.
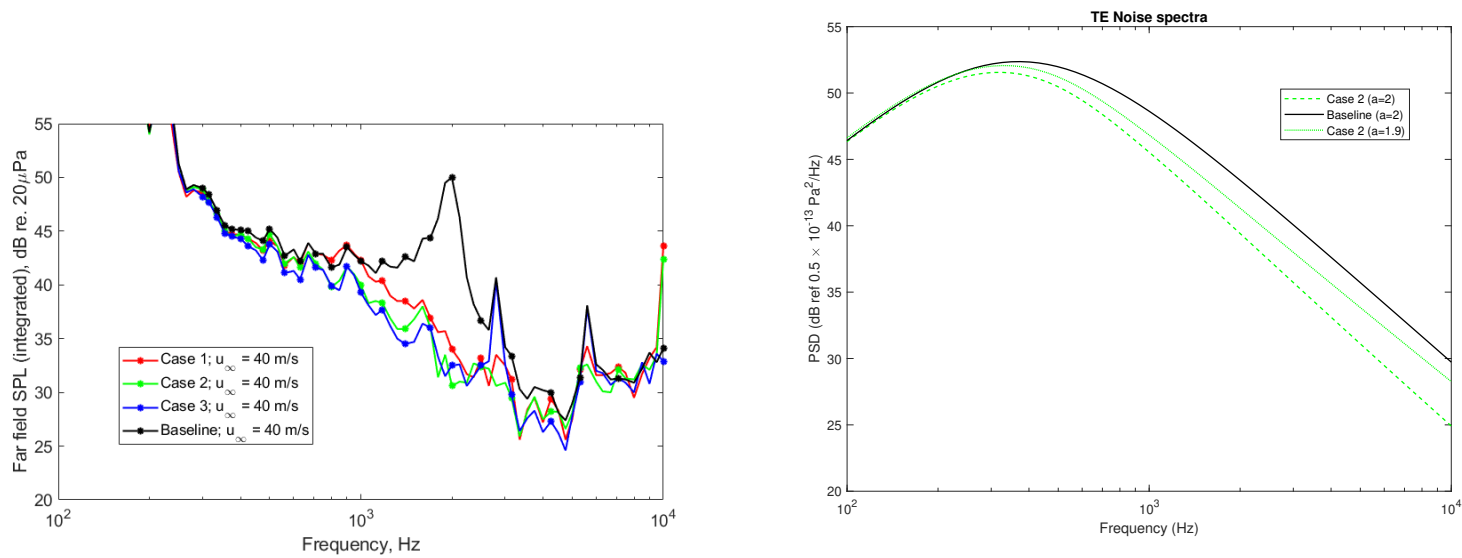

Fig. 14 Left: integrated far-field noise SPL levels around the trailing edge of the serrations at $40 \mathrm{~m} / \mathrm{s}$ freestream velocity. Right: Theoretical predictions using the altered Chase spectrum (14).

$\phi_{v v}$, whereas $a<2$ indicates a slower decay rate, as observed for the serration.

Figure 14 illustrates the effect of altering the theoretical decay rate on the far-field predicted noise for Case 2, again side by side to the experimental measurements. By using an altered spectrum we see a much better comparison to the experimental measurements, both in the overall level of noise reduction and in the decreasing noise reduction as frequency increases. 


\section{Conclusions}

This paper has considered the component parts of a theoretical model for predicting the far-field noise from a serrated trailing edge; one part is the analytically derived scattering of a single-frequency wall pressure gust, whilst the second part is a wavenumber-frequency spectrum which must be integrated over to analyse a fully turbulent flow. Attention has been given to this second component, and how serrations themselves may affect the properties of turbulence in the boundary layer.

Through targeted experimental tests, aspects of the wavenumber-frequency spectrum for a serrated edge have been measured and compared to that for a baseline straight edge. Different locations of the serration exhibit different vertical velocity frequency spectra which when input to a theoretical model would of course confer different predictions. Of particular note was the decay rate in $\phi_{v v}(f)$ at high frequencies, which was seen to be reduced in the case of the serration compared to the baseline. A simple adaptation to the Chase-type empirical model that reflects an altered high-frequency decay rate then showed much closer agreement to the experimentally measured far-field noise.

We conclude that the most likely reason behind the lack of agreement between theoretical and experimental measurements for far-field noise is therefore down to the wavenumber-frequency spectra being altered by the serrations thus incorrectly input to the serrated noise prediction model. It is a topic of future work to determine the correct alteration to an empirical model to best represent the differences observed in the experimental spectra across a range of different serrations, however initial results indicate the decay rate parameter is the key feature to be adapted.

\section{Acknowledgments}

L. J. Ayton acknowledges support from EPSRC Early Career Fellowship EP/P015980/1 and P. Chaitanya from the Royal Academy of Engineering (RF/201819/18/194).

\section{References}

[1] Graham, R. R. 1934 The silent flight of owls. Journal of the Royal Aeronautical Society 38, 837-843.

[2] Lilley G. M. 1998 A study of the silent flight of the owl. 4th AIAA/CEAS Aeroacoustics Conference, Toulouse, AIAA $1998-2340$.

[3] Jaworski, J.W. \& Peake, N. 2013 Aerodynamic noise from a poroelastic edge with implications for the silent flight of owls. Journal of Fluid Mechanics 723, 456-479.

[4] Gruber, M. 2012 Airfoil noise reduction by edge treatments. Ph.D Thesis, University of Southampton.

[5] Moreau, D. J. \& Doolan, C. J. 2013 Noise-Reduction Mechanism of a Flat-Plate Serrated Trailing Edge. AIAA Journal 51, $2513-2522$

[6] Sanjose, M., Meon, C., Masson, V., \& Moreau S. 2014 Direct numerical simulation of acoustic reduction using serrated trailing-edge on an isolated airfoil, 20th AIAA/CEAS Aeroacoustics 2324.

[7] van der Velden, W. C., Avallone, F. \& Ragni, D. 2017 Numerical analysis of noise reduction mechanisms of serrated trailing edges under zero lift condition. 23rd AIAA/CEAS Aeroacoustics Conference, AIAA, 2017-4173.

[8] Ayton, L. J. 2018 Analytic solution for aerodynamic noise generated by plates with spanwise-varying trailing edges. Journal of Fluid Mechanics 849 448-466.

[9] Avallone, F., van der Velden, W. C. P. and Ragni, D. 2017 Benefits of curved serrations on broadband trailing-edge noise reduction. Journal of Sound and Vibration 400 167-177.

[10] Chaitanya, P., Joseph, P. F., Narayanan, S., Vanderwel, C., Turner, J., Kim, J.-W. \& Ganapathisubramani, B. 2017 Performance and mechanism of sinusoidal leading edge serrations for the reductions of aerofoil interaction noise. Journal of Fluid Mechanics 818 435-464.

[11] Biedermann, T. M., Chong, T. P., Kameier, F. \& Paschereit, C. O. 2017 Statistical empirical modeling of airfoil noise subjected to leading-edge serrations. AIAA Journal 55 3128-3142.

[12] Chaitanya, P., Joseph, P. F. \& Ayton, L. J. 2018 On the superior performance of leading edge slits over serrations for the reduction of aerofoil interaction noise. 24th AIAA/CEAS Aeroacoustics AIAA 2018-3121.

[13] Ayton, L. J. \& Chaitanya, P. 2019 An Analytical and Experimental Investigation of Aerofoil-Turbulence Interaction Noise for Plates with Spanwise-Varying Leading Edges. Journal of Fluid Mechanics 865 137-168. 
[14] Chong, T. P., Vathylakis, A., Joseph, P. F. \& Gurber, M. 2016 Self-Noise Produced by an Airfoil with Nonflat Plate Trailing-Edge Serrations. AIAA Journal 51, 2665-2677.

[15] Letica, S. \& Alexander, W. N. 2021 Understanding the Impact of a Serrated Trailing Edge on the Unsteady Hydrodynamic Field, Journal of Aerospace Engineering 34, 04021045.

[16] Moreau, S., Sanjose, M, Lyu, B. \& Ayton, L. J. 2019 Analytical, numerical and experimental investigation of trailing-edge noise reduction on a Controlled Diffusion airfoil with serrations. 25th AIAA/CEAS Aeroacoustics AIAA 2019-2450.

[17] Howe, M. S. 1991 Aerodynamic noise of a serrated trailing edge. Journal of Fluids and Structures 5, 33-45.

[18] Chase, D. M. 1987 The character of the turbulent wall pressure spectrum at subconvective wavenumbers and a suggested comprehensive model. Journal of Sound and Vibration 112, 125-147.

[19] Devenport, W. J., Burdisso, R., Borgoltz, A., Ravetta, P., Barone, M., Brown, K. A., Morton, M. A. 2013 The Kevlar-walled anechoic wind tunnel. Journal of Sound and Vibration 332, 3971-3991.

[20] Brown, K. A., Devenport, W. J., Borgoltz, A. 2019 Aircraft noise generation and assessment: exploitation of hybrid anechoic wind tunnels for aeroacoustic and aerodynamic measurements. CEAS Aeronautical Journal 10(1), 251-266.

[21] Schewe, G. 1983 On the structure and resolution of wall-pressure fluctuations associated with turbulent boundary-layer flow. Journal of Fluid Mechanics 134, 311-328.

[22] Kline, S. J. \& McClintock, F. A. 1953 Describing uncertainties in single-sample experiments. Mechanical Engineering 75(1), 3-8.

[23] Fischer, A., Bertagnolio, F. \& Madsen, H. A. 2016 Improvement of TNO type trailing edge noise models. ISROMAC 2016 